\title{
研究 通讯
}

\section{权为 $3 / 2$ 的 Eisenstein 级数}

在群 $\Gamma_{0}(N)$ 上对应特征 $\omega$ 的模形式空间中, 歧 点型模形式的正交补空间称为 Eisenstein 空间. 当 权为整数或 $\geqslant \frac{5}{2}$ 的半整数时, 熟知这一子空间是由 Eisenstein 级数生成的. 于是尚需研究权为 $\frac{1}{2}$ 和 $\frac{3}{2}$ 两个情况. 1976 年 Serre 和 Stark 证明了权为 $\frac{1}{2}$ 时该结论成立. 现在我们证明了当权为 $\frac{3}{2}$ 时也成 立. 在证明中我们具体地构造了该空间的一组基. 以 $N=4 D$ ( $D$ 为无平方因子的正奇数)， $\omega$ 为恒 为 1 的特征为例,该空间的一组基为

$$
\begin{aligned}
& g(4 D, 4 D)=1-4 \pi(1+i) \sum_{n=1}^{\infty} \lambda(n, 4 D) \\
& \times\left(A(2, n)-\frac{1-i}{4}\right) \\
& \quad \times \prod_{p \mid D}\left(A(p, n)-p^{-1}\right) n^{1 / 2} e(n z), \\
& g(4 m, 4 D)=-4 \pi(1+i) \sum_{n=1}^{\infty} \lambda(n, 4 D) \\
& \quad \times\left(A(2, n)-\frac{1-i}{4}\right) \\
& \quad \times \prod_{p \mid m}\left(A(p, n)-p^{-1}\right) n^{1 / 2} e(n z), \\
& \quad \times \prod_{p \mid m}\left(A(p, n)-p^{-1}\right) n^{1 / 2} e(n z),
\end{aligned}
$$

$$
(m \mid D, m \neq 1) \text {, }
$$

这里(设 $\left.p^{h(p)} \| n\right)$

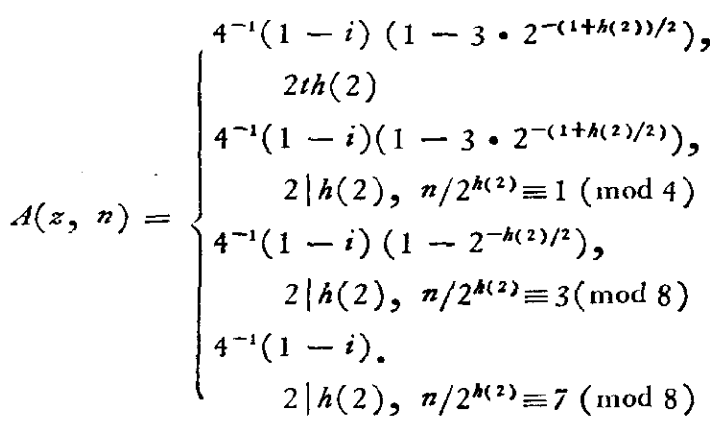

当 $p \neq 2$ 时

$$
A(p, n)=\left\{\begin{array}{c}
p^{-1}-(1+p) p^{-(3+h(p)) / 2} \\
2 t h(p) . \\
p^{-1}-2 p^{-1-h(p) / 2} \\
2 \mid h(p),\left(\frac{-n / p^{h(p)}}{p}\right)=-1, \\
p^{-1} \cdot \quad 2 \mid h(p),\left(\frac{-n / p^{h(p)}}{p}\right)=1 .
\end{array}\right.
$$$$
\lambda(n, 4 D)=\frac{L_{4 D}\left(1,\left(\frac{-n}{\cdot}\right)\right)}{L_{A D}(2, i d \cdot)} \sum \mu(a)\left(\frac{-n}{a}\right)
$$$$
\times(a b)^{-1},
$$

对所有适合 $(a b, 4 D)=1,(a b)^{2} \mid n$ 的 $a, b$ 求和, $\left(\frac{-n}{a}\right)$ 是 $\mathrm{Jacobi}$ 符号.

\section{裴定一}

(中国科学院应用数学所)

\section{富里埃级数论中一些定理的等价性}

1. 设 $x \cdot g(x) \in L(0, \pi)$,

$b_{n}(g)=\frac{2}{\pi} \int_{0}^{\pi} g(x) \sin n x d x \quad(n=1,2, \cdots)$.

杨义群改进了 Boas 的定理, 证得

$$
\text { 定理 } \mathbf{A} \text { 级数 } \sum_{1}^{\infty} n^{-2} b_{n}(g)
$$

收敛的充要条件是 $\frac{1}{x} \int_{0}^{x} t \cdot g(t) d t \in C L$, 这里 $f(x) \times$ $\in C L$ 表示 $f$ 的柯西一勒贝格积分存在,即 $\lim _{\varepsilon \rightarrow 0+} \int_{\varepsilon}^{*}$ - $f(x) d x$ 存在.

另一方面, Hardy-Littlewood 早就证明过如下 的

\section{定理 B 设}

$f \in L(0, \pi), S[f]=\frac{a_{0}}{2}+\sum_{1}^{\infty}\left(a_{n} \cos n x\right.$

$$
\left.+b_{n} \sin n x\right) \text {, }
$$


那么级数 $\sum_{1}^{\infty} n^{-1} a_{n}$ 收敛的充要条件是 $\operatorname{ctg} \frac{x}{2} \int_{-x}^{\lambda} f(t)$. $d t \in C L$.

\section{本文第一个目的是证明}

定理 1 定理 $A$ 与定理 B 是等价的.

2. Hardy 在 $a_{n} \downarrow 0$ 的假设下, 指出: $a_{n} \simeq \frac{1}{n}$ 含有 $\sum_{0}^{\infty} a_{n} \cos n x \simeq \ln \frac{1}{x}(x \rightarrow 0+)$. 杨义群最近证 明了

$$
\begin{gathered}
\text { 定理 } \alpha \quad \text { 当 } a_{n} \downarrow 0 \text { 时, } \\
\sum_{0}^{\infty} a_{n} \cos n x \simeq \ln \frac{1}{x}(x \rightarrow 0+)
\end{gathered}
$$

的充要条件是

$$
\sum_{k=1}^{n} a_{k} \simeq \ln n \quad(n \rightarrow \infty) .
$$

另方面, Heywood 在 $b_{n} \downarrow 0$ 的假设下，指出： $b_{n}=\frac{1}{n^{2}}$ 含有 $\sum_{1}^{\infty} b_{x} \sin n x \simeq x \ln \frac{1}{x}(x \rightarrow 0+)$. 杨义 群证明

定理 $\beta$ 假如 $\sum_{1}^{\infty} b_{n} \sin n x$ 是 Fouries 级数, $\dot{b}_{n} \geqslant 0$, 那么 $\sum_{1}^{\infty} b_{n} \sin n x \simeq x \ln \frac{1}{x}(x \rightarrow 0+)$ 的充要 条件是 $\sum_{1}^{n} k b_{k} \simeq \ln n$.

本文第二个目的是证明

定理 2 定理 $\alpha$ 和定理 $\beta$ 是等价的.

以上一些定理的等价性不仅刻划了它们的内在 联系,而且也是相应定理的一种简单证明.

\section{王斯雷 \\ (杭州大学)}

\section{关于伪球面上子流形的高斯映照}

设 $M$ 是一个 $n$ 维黎曼流形. 最近, 陈咸平证得: 等距浸入 $f: M^{c} \rightarrow S^{n+p}$ 的高斯映照 $g: M \hookrightarrow G_{n+1, p}$ 是调 和的, 当且仅当 $f$ 是极小浸入, 这里 $S^{n+p}$ 是 $(n+p)$ 维球面, $G_{n+1, p}$ 是 Grassmann 流形。彭家贵未加证 明地指出,对于伪球面上子流形的高斯映照,类似的 命题也成立. 本文证实了这个猜测.

设 $H^{n+p}$ 是 $(n+p)$ 维伪球面, $Q$ 表示 $H^{n+p}$ 中 一切 $n$ 维全测地子空间的集合. 设 $\tilde{f}: M \hookrightarrow H^{n+p}$ 是一
个等距浸入. 那么, 由 Obata 定义的 $\tilde{f}$ 的高斯映照 是 $\tilde{g}: M \hookrightarrow Q$, 其中对于任一点 $p \in M, \tilde{g}(p)$ 是在点 $\tilde{f}(p)$ 与 $\tilde{f}(M)$ 相切的 $n$ 维全测地子空间.

\section{我们证明了下列}

定理 等距浸入 $\tilde{f}: M \hookrightarrow H^{n+p}$ 的高斯胦照 $\tilde{g}$ : $M \hookrightarrow Q Q$ 是调和的，当且仅当 $\tilde{f}$ 是极小浸入.

沈一兵

(杭州大学)

\section{含 $\boldsymbol{\nu}$-基座本原环的两个结果}

许永华引进了含 $\nu$-基座本原环的概念, 建立了 $\nu$-结构定理（数学学报, 1979, 22:5). 对含 $\nu$-基座 的本原环, 我们得到以下两个结果.

定理 1 设 $R$ 是含 $\boldsymbol{\nu}$-基座 $\sigma_{\boldsymbol{y}}$ 的本原环, 对任 何 $\lambda<\nu, R$ 必是含有 $\lambda$-基座的本原环.

因为本原环的 $O$ - 基座概念与通常基座概念一 致, 故由定理 1 知, 任何含 $\nu$-基座的本原环, 必是通 常意义下的含非零基座的本原环.

若 $R$ 是含 $\nu$-基座的本原环, 由 $\boldsymbol{\nu}$-结构定理, 存在 除环 $F$ 上的对偶空间 $\left(A, A^{\prime}\right)$, 它是 $\nu-A^{\prime}$-可和的, $A^{\prime}$
是 $\boldsymbol{\nu}$ 完全的, 并且 $R$ 与 $L_{A^{\prime}}^{(\nu)}(A)$ 中含 $T_{A^{\prime}}^{(\boldsymbol{y})}(A)$ 的子 环问构, 其中 $L_{A^{\prime}}^{\left(\nu^{\prime}\right)}(A), T_{A^{\prime}}^{(\nu)}(A)$ 分别表示 $A$ 在 $\nu-A$ 拓扑下所有强连续线性变换环和所有有限秩强连续 线性变换环. 我们简称除环 $F$ 上的对偶空间 ( $A$, $\left.A^{\prime}\right)$ 为在 $R$ 的 $\boldsymbol{\nu}$-结构定理中的对偶空间. $R$ 是含 $\nu-$ 基座的本原环， $R$ 也就是通常意义下的含非零基座 的本原环, 由通常结构定理, 存在除环 $F^{\prime}$ 上的对偶 空间 $\left(B, B^{\prime}\right)$, 并且 $R$ 与 $\mathfrak{L}_{B^{\prime}}(B)$ 中含 $\mathfrak{F}_{B^{\prime}}(B)$ 的子环 同构, 其中 $\mathfrak{L}_{B^{\prime}}(B), \mathfrak{F}_{B^{\prime}}(B)$ 分别表示 $B$ 在通常有限 拓扑下所有连续线性变换环和所有有限秩连续线性 